\title{
Flares in Rheumatoid Arthritis Patients with Low Disease Activity: Predictability and Association with Worse Clinical Outcomes
}

\author{
Katie Bechman (1), Lieke Tweehuysen, Toby Garrood, David L. Scott, Andrew P. Cope (1), \\ James B. Galloway ${ }^{(1)}$, and Margaret H.Y. Ma
}

ABSTRACT. Objective. To investigate predictors of flare in rheumatoid arthritis (RA) patients with low disease
activity (LDA) and to evaluate the effect of flare on 12-month clinical outcomes.
Methods. Patients with RA who were taking disease-modifying antirheumatic drugs and had a stable
28-joint count Disease Activity Score (DAS28) $<3.2$ were eligible for inclusion. At baseline and every
3 months, clinical (DAS28), functional [Health Assessment Questionnaire-Disability Index
(HAQ-DI), EQ-5D, Functional Assessment of Chronic Illness Therapy Fatigue scale (FACIT-F),
Medical Outcomes Study Short Form-36 (SF-36)], serum biomarkers [multibiomarker disease activity
(MBDA) score, calprotectin, CXCL10], and imaging data were collected. Flare was defined as an
increase in DAS28 compared with baseline of $>1.2$, or $>0.6$ if concurrent DAS28 $\geq 3.2$. Cox
regression analyses were used to identify baseline predictors of flare. Biomarkers were cross-
sectionally correlated at time of flare. Linear regressions were performed to compare clinical outcomes
after 1 year.

Results. Of 152 patients, $46(30 \%)$ experienced a flare. Functional disability at baseline was associated with flare: HAQ-DI had an unadjusted HR 1.82 (95\% CI 1.20-2.72) and EQ-5D had HR 0.20 (95\% CI 0.07-0.57). In multivariate analyses, only HAQ-DI remained a significant independent predictor of flare (HR 1.76, 95\% CI 1.05-2.93). At time of flare, DAS28 and its components significantly correlated with MBDA and calprotectin, but correlation coefficients were low at 0.52 and 0.49 , respectively. Two-thirds of flares were not associated with a rise in biomarkers. Patients who flared had significantly worse outcomes at 12 months (HAQ-DI, EQ-5D, FACIT-F, SF-36, and radiographic progression).

Conclusion. Flares occur frequently in RA patients with LDA and are associated with worse disease activity, quality of life, and radiographic progression. Higher baseline HAQ-DI was modestly predictive of flare, while biomarker correlation at the time of flare suggests a noninflammatory component in a majority of events. (First Release September 1 2018; J Rheumatol 2018;45:1515-21; doi:10.3899/jrheum.171375)

Key Indexing Terms: RHEUMATOID ARTHRITIS SERUM BIOMARKERS FLARE

From the Academic Department of Rheumatology, Kings College London, London; Department of Rheumatology, Guy's and St Thomas' UK National Health Service (NHS) Foundation Trust, London, UK; Department of Rheumatology, Sint Maartenskliniek, Nijmegen, the Netherlands.

This report represents independent research by Katie Bechman, partly funded by the UK National Institute for Health Research (NIHR) Biomedical Research Centre at Guy's and St Thomas' NHS Foundation Trust and King's College London. Margaret Ma's work was funded by the NIHR (DRF-2009-02-86 to M.H.Y. Ma).

K. Bechman, $M B C h B, B S c$, Rheumatology Registrar and Clinical Research Fellow, Academic Department of Rheumatology, Kings College London; L. Tweehuysen, MD, Rheumatology Registrar and Clinical Research Fellow, Department of Rheumatology, Sint Maartenskliniek; T. Garrood, MBBS, MRCP, MSc, PhD, Consultant Rheumatologist, Department of Rheumatology, Guy's and St Thomas' NHS Foundation Trust; D.L.Scott, BSc, MD, FRCP, Professor of Rheumatology, Academic Department of Rheumatology, Kings College London; A.P. Cope, BSc, MBBS, PhD, FRCP, FHEA, Professor of Rheumatology, Academic Department of Rheumatology, Kings College London; J.B. Galloway, $M B C h B, M S c, C H P, M R C P, P h D$, Consultant Rheumatologist/Senior Lecturer, Academic Department of Rheumatology, Kings College London; M.H. Ma, MBBS, BSc, MRCP, MSc, PhD, Consultant Rheumatologist,
Academic Department of Rheumatology, Kings College London. Address correspondence to K. Bechman, Academic Rheumatology Department, Third Floor, Room 3.46, Weston Education Centre, King's College London, London SE5 9RJ,UK.E-mail: katie.bechman@kcl.ac.uk Accepted for publication April 27, 2018.

Guidelines for the treatment of rheumatoid arthritis (RA) have emphasized a "treat-to-target" approach with the explicit aim of low disease activity (LDA) states ${ }^{1,2}$. However, disease activity in RA can fluctuate. Episodic worsening of disease activity, described as "flare," is common. Flare was originally defined by the Outcome Measures in Rheumatology Clinical Trials (OMERACT 9) group as a cluster of symptoms of sufficient duration and intensity to require initiation, change, or increase in therapy ${ }^{3}$. These definitions focused on the more severe end of the flare continuum for evaluation of flares in randomized controlled trials. In daily practice, flare can vary in duration, intensity,

Personal non-commercial use only. The Journal of Rheumatology Copyright (c) 2018. All rights reserved. 
frequency, and manageability ${ }^{4}$, with about half of patients in remission experiencing a disease flare within 2 years ${ }^{5}$. This has important clinical implications because flares in patients with apparent LDA states are associated with radiographic progression $^{6,7}$, functional deterioration ${ }^{7}$, and worsening cardiovascular comorbidity ${ }^{8}$.

Predicting flare is therefore of direct relevance to clinical practice. Saleem, et al demonstrated that functional disability [Health Assessment Questionnaire-Disability Index (HAQ-DI)] and power Doppler ultrasound (PDUS) positivity at baseline were independently associated with flare in patients with RA in remission ${ }^{9}$. Further, a previous metaanalysis revealed an association between PDUS positivity and flare in RA patients in remission ${ }^{10}$.

The finding of PD positivity despite clinical remission provides evidence that flares may be related to incomplete suppression of inflammation. Based on this hypothesis, serum biomarkers may detect subclinical disease activity and consequently predict flare. In contrast to ultrasound (US), biomarkers may have smaller measurement error and may be less operator-dependent, costly, and time-consuming. In recent years, the predictive values of the multibiomarker disease activity (MBDA) score, calprotectin (S100A8/A9), and CXCL10 for treatment response in RA have been investigated. In the DRESS study, baseline MBDA score was predictive of flare and major flare in patients with LDA who did not taper treatment (usual care group) ${ }^{11}$. To our knowledge, calprotectin and CXCL10 have not been investigated as predictors of flare in disease-modifying antirheumatic drug (DMARD)-treated patients with RA in an LDA state. Calprotectin was found to be more strongly associated with US-detected synovitis than erythrocyte sedimentation rate (ESR) or C-reactive protein $(\mathrm{CRP})^{12}$, and baseline calprotectin appeared to be predictive of clinical response to methotrexate ${ }^{13}$. However, its predictive role as a marker of response to biologic DMARD is conflicting ${ }^{14,15}$. CXCL10 was correlated with multiple disease activity measures in early $\mathrm{RA}^{16}$, while elevated baseline levels of CXCL10 were associated with favorable response to tumor necrosis factor (TNF) inhibitor therapy in $\mathrm{RA}^{10}$.

The aims of our study were 3 -fold. First, we aimed to describe the frequency of flares in a cohort of prospective patients with RA in stable LDA states (including remission) over 1 year. Second, we aimed to examine the predictive value of a wide range of biomarkers (including clinical, functional, serum, and imaging variables) for flare. And third, we aimed to evaluate the effect of flare in RA patients with LDA states.

\section{MATERIALS AND METHODS}

Study design and patients. The REMIRA study is a prospective cohort study investigating RA patients with stable LDA states including clinical remission. Clinical outcomes have been reported recently ${ }^{17}$. Adult patients with RA were eligible for inclusion if they were diagnosed according to the 1987 revised American College of Rheumatology criteria with a disease duration $<10$ years, had stable DMARD treatment for $>6$ months, and 28 -joint count Disease Activity Score (DAS28) $<3.2$ for at least 1 month apart. Three centers across South London participated: Guy's and St. Thomas' Hospital, King's College Hospital, and University Hospital Lewisham National Health Service Foundation Trusts. Patients were managed as part of routine care. The study was approved by the local ethics committee and conducted according to the guidelines of the Declaration of Helsinki (REC:09/H0803/154). Written informed consent was obtained from all patients.

Clinical assessments. At baseline, demographic, disease and treatment characteristics were collected. Clinical assessments were carried out every 3 months for 1 year and included pain and fatigue (both on visual analog scale 0-100), DAS28, CRP, and ESR. Questionnaires were used to assess function and quality of life: HAQ-DI, EQ-5D-3L, Medical Outcomes Study Short Form-36 [SF-36; including physical component score (PCS) and mental component score (MCS)] and Functional Assessment of Chronic Illness Therapy Fatigue scale (FACIT-F). Flare was defined according to previously validated criteria: a DAS2 8 increase of $>1.2$ compared with baseline or a DAS28 increase of $>0.6$ compared with baseline and concurrent DAS $28 \geq 3.2^{18}$. For patients with multiple flares, only the first flare was considered in the analyses.

Serum biomarker measurements. Serum samples were obtained at each timepoint and stored at $-80^{\circ} \mathrm{C}$ until being shipped frozen to the Crescendo Bioscience Clinical Laboratory (South San Francisco, California, USA) for MBDA score, calprotectin, and CXCL10 measurement. The MBDA test (Vectra DA, Crescendo Bioscience) combines the serum concentrations of 12 protein biomarkers [interleukin 6 , TNF receptor type I, vascular cell adhesion molecule 1, epidermal growth factor, vascular endothelial growth factor A, YKL-40, matrix metalloproteinase 1, matrix metalloproteinase 3 , CRP, serum amyloid A (SAA), leptin, and resistin] in an algorithm to provide a score that quantifies RA disease activity. The scores are on a scale of 1 to 100 with validated categories for low $(\leq 30)$, moderate (30-44), and high disease activity $(>44)^{19}$. Calprotectin and CXCL10 were measured by ELISA (Buhlmann MRP 8/14 ELISA Product Code EK-MRP8/14m; R\&D Systems Human CXCL10/IP-10 Quantikine ELISA Product Code DIP100).

Imaging assessments. Ultrasonography of hands and wrists, and conventional radiographs of hands and feet were carried out at baseline and 12 months. Erosive progression was defined as new or larger erosions over 1 year on radiographs. All sonographic assessments were performed using high-sensitivity US equipment (GE Logiq 9) with a 2-dimensional M12L transducer. A single experienced sonographer (TG), blinded to clinical or laboratory data, scanned 10 metacarpophalangeal joints and 2 wrists from a dorsal aspect for greyscale US (GSUS) synovial hypertrophy and intraarticular PDUS signals ${ }^{20}$. GSUS and PDUS were graded on a scale of 0 to 3 using a validated semiquantitative scoring system ${ }^{21}$. The composite GSUS and PDUS scores were the sum scores of the 12 individual joints.

Statistical analysis. Descriptive statistics were provided with mean ( \pm SD), median (interquartile range; IQR), or frequencies, depending on data distribution. Cross-sectional correlations between all measurements (biomarkers and DAS28 components) at time of flare were assessed by Spearman's correlation coefficient (rs), and interpreted according to commonly used classification: very weak ( $\mathrm{rs}<0.20)$, weak $(\mathrm{rs}=0.20-0.39)$, moderate $(\mathrm{rs}=0.40-0.59)$, strong $(\mathrm{rs}=0.60-0.79)$, and very strong $(\mathrm{rs}>0.80)$ correlation $^{22}$.

To identify predictors of time to flare, we performed univariate Cox regression, in which time to flare was the dependent variable, and clinical, functional, serum, and imaging measurements the independent variables. Multivariate analyses were performed to identify factors that were independently associated with flare, adjusting for age, sex, DAS28, visual analog scale (VAS) pain, CRP, ESR, and US scores (for HAQ model only), and MBDA score (for EQ-5D model only).

Linear regression was used to determine the effect of flare on 12-month clinical outcomes (i.e., disease activity and functional status). A multivariate linear regression model was applied adjusting for baseline age, sex, disease

Personal non-commercial use only. The Journal of Rheumatology Copyright $\odot$ (2018. All rights reserved 
duration, erosive status, baseline DAS28, HAQ, and baseline variable of interest. A p value $\leq 0.05$ was regarded as significant. Because this was an exploratory study, no correction for multiple hypothesis testing was performed. Missing data were addressed using a multiple imputation module (Supplementary Data 1, available from the authors on request). All analyses were performed with STATA 14.1 statistical software.

\section{RESULTS}

Patient characteristics. In total, 152 patients were enrolled in the REMIRA study. Baseline characteristics are depicted in Table 1 . The majority of patients were receiving DMARD monotherapy $(n=69 ; 45 \%)$ and the median disease duration was 3 (IQR 2-6) years. Ninety-seven patients (66\%) fulfilled DAS28 remission criteria (DAS28 <2.6). All patients had

Table 1. Patient characteristics.

\begin{tabular}{|c|c|}
\hline Characteristics & Patients, $\mathrm{n}=152$ \\
\hline Age, yrs, mean (SD) & $57(14)$ \\
\hline Female sex, n (\%) & $101(66)$ \\
\hline Disease duration, yrs & $3(2-6)$ \\
\hline \multicolumn{2}{|l|}{ Treatment, n (\%) } \\
\hline csDMARD monotherapy & $69(45)$ \\
\hline csDMARD combination therapy & $59(39)$ \\
\hline bDMARD therapy & $24(16)$ \\
\hline Prednisolone & $3(2)$ \\
\hline Seropositive (RF and/or ACPA), n (\%) & $103 / 137(75)$ \\
\hline Erosive, n (\%) & $67(45)$ \\
\hline TJC28 & $0(0-1)$ \\
\hline SJC28 & $0(0-2)$ \\
\hline PtGA, 0-100 mm & $19(10-36)$ \\
\hline ESR & $7(4-13)$ \\
\hline $\mathrm{CRP}, \mathrm{mg} / \mathrm{l}$ & $5(1-31)$ \\
\hline DAS28-ESR, mean (SD) & $2.1(0.9)$ \\
\hline DAS28 remission, $\mathrm{n}(\%)$ & $97 / 148(66)$ \\
\hline VAS pain, $0-100 \mathrm{~mm}$ & $15(3-34)$ \\
\hline HAQ-DI & $0.25(0-0.86)$ \\
\hline EQ-5D & $0.76(0.69-1.00)$ \\
\hline SF-36 PCS, mean (SD) & $46(11)$ \\
\hline SF-36 MCS, mean (SD) & $51(10)$ \\
\hline FACIT-F & $42(34-47)$ \\
\hline MBDA score, $1-100$ & $31(18-39)$ \\
\hline Calprotectin, ng/ml & $2358(1487-3358)$ \\
\hline CXCL10, pg/ml & $198(143-291)$ \\
\hline No. $(\%)$ patients with GSUS $>0$ & 104/104 (100) \\
\hline Total GSUS score, /36 & $12(8-14)$ \\
\hline No. $(\%)$ patients with PDUS $>0$ & $93(90)$ \\
\hline Total PDUS score, /36 & $2(1-4)$ \\
\hline
\end{tabular}

Data are median (IQR) unless otherwise specified. IQR: interquartile range; csDMARD: conventional synthetic disease-modifying antirheumatic drug; bDMARD: biologic DMARD; RF: rheumatoid factor; ACPA: anticitrullinated protein antibody; TJC28: tender joint count at 28 joints; SJC28: swollen joint count at 28 joints; PtGA: patient's global assessment; CRP: C-reactive protein; ESR: erythrocyte sedimentation rate; DAS28: 28-joint count Disease Activity Score; VAS: visual analog scale; HAQ-DI: Health Assessment Questionnaire-Disability Index; SF-36 PCS: Medical Outcomes Study Short Form-36 physical component summary; SF-36 MCS: Medical Outcomes Study Short Form-36 mental component summary; FACIT-F: Functional Assessment of Chronic Illness Therapy Fatigue scale; MBDA: multibiomarker disease activity; GSUS: greyscale ultrasound synovial hypertrophy; PDUS: intraarticular power Doppler ultrasound signals. synovial hypertrophy (GSUS > 1) and $90 \%$ had detectable PDUS activity at baseline.

Characteristics of flare. Forty-six patients (30\%) experienced at least 1 flare. Twelve patients had first flare by 3 months, 10 by 6 months, 11 by 9 months, and 13 by 12 months. Seventeen patients experienced multiple flares; 11 patients flared at 2 visits, 5 patients at 3 visits, and 1 patient at all 4 visits after baseline. When limiting the cohort to patients who were in remission defined by DAS28 $<2.6$ at baseline, 24 patients of a total $97(25 \%)$ experienced at least 1 flare.

Serum biomarkers at time of flare. There were 70 individual flare events. Seventeen percent $(n=12)$ of flares were driven solely by increases in patient's global assessment (PtGA) and tender joint count (TJC), without any increase in swollen joint count (SJC) or ESR.

In total, $33 \%$ of flares $(n=23)$ had a concurrent high MBDA score $(>44)$, while $13 \%(n=44)$ of visits without flare had a high MBDA score. The levels of ESR, CRP, MBDA score, and calprotectin were significantly higher at flare visits than at nonflare visits [median ESR $14 \mathrm{~mm} / \mathrm{h}$ (IQR 5-23) vs $6 \mathrm{~mm} / \mathrm{h}$ (3-12); CRP $5 \mathrm{mg} / \mathrm{l}$ (5-9) vs $5 \mathrm{mg} / \mathrm{l}$ (5-5); MBDA 38 (25-50) vs 28 (18-38); and calprotectin 2916 $\mathrm{ng} / \mathrm{ml}(2002-4186)$ vs $2377 \mathrm{ng} / \mathrm{ml}$ (1504-3358)].

DAS28 significantly correlated with MBDA score $\left(r_{s}=0.5, p=0.0002\right)$ at time of flare. The $r_{s}$ of 0.5 suggests that the MBDA values explain only $25 \%$ of the variation in DAS28. The correlation of MBDA was stronger with the components ESR and SJC, and were nonsignificant for TJC and PtGA. Similar findings were seen for calprotectin ( $\mathrm{rs}=0.49, \mathrm{p}=0.0007)$. CXCL10 did not correlate with DAS28 or its components at time of flare (Supplementary Table 1, available from the authors on request).

Prediction of flare. Univariate Cox regression showed that several baseline characteristics were associated with flare (DAS28, ESR, CRP, PtGA, VAS pain, HAQ-DI, and EQ-5D; Figure 1 and Supplementary Tables 2 and 3, available from the authors on request). The strongest magnitude of association was seen with HAQ-DI and EQ-5D. Baseline US synovitis (GSUS or PDUS) and mental health (using the SF-36 MCS) were not associated with flare. Baseline MBDA scores were also not predictive of flare, although a sensitivity analysis limited to flares with a rise in MBDA score to $>44$ (high disease activity) did show a relationship between baseline MBDA value and flare risk, with each unit rise in baseline MBDA score associated with a $7 \%$ increase in flare risk $(1.07,95 \%$ CI 1.02-1.11; p $=0.005$; Supplementary Tables 4 and 5). Analyzing each component of the MBDA score identified SAA, leptin, and high-sensitivity CRP as the strongest predictors of flare. The remaining 9 components of the MBDA score did not individually predict flare.

The imputation model confirmed the association between flare and baseline HAQ-DI and EQ-5D but did not demonstrate any other associations. In multivariate analyses, only baseline HAQ-DI remained a significant independent

Personal non-commercial use only. The Journal of Rheumatology Copyright $\odot$ 2018. All rights reserved 


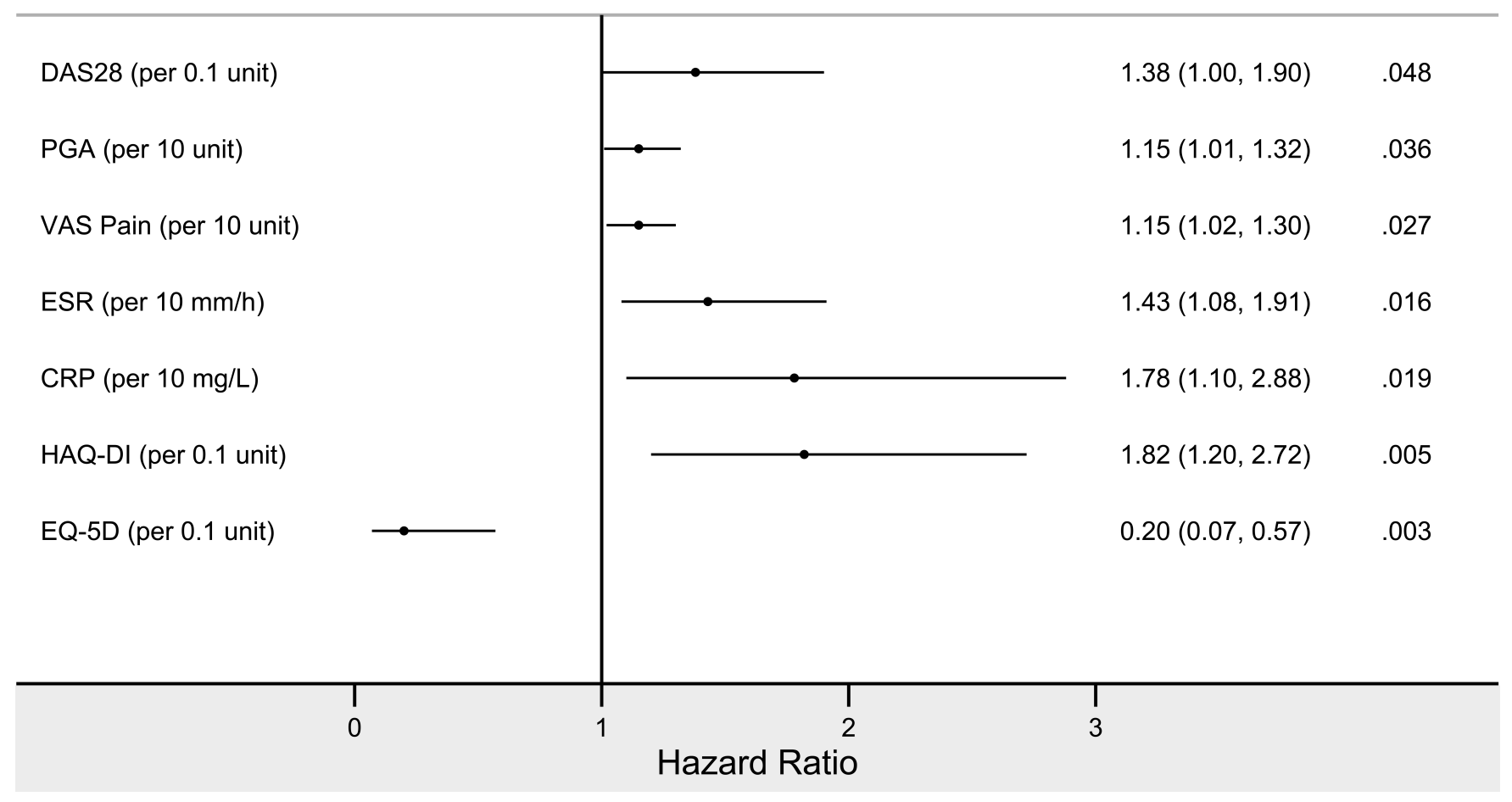

Figure 1. Univariate analyses of prediction of flare with baseline variables. DAS28: 28-joint count Disease Activity Score; PGA: patient's global assessment; VAS: visual analog scale; ESR: erythrocyte sedimentation rate; CRP: C-reactive protein; HAQ-DI: Health Assessment Questionnaire-Disability Index.

predictor of flare (HR 1.76, 95\% CI 1.05-2.93, $\mathrm{p}=0.03$; Supplementary Table 2, available from the authors on request).

Outcomes in flare versus sustained remission group. Adjusting for baseline values, patients who had a flare experienced significantly worse clinical outcomes at 12 months than patients in sustained remission, reflected by higher disease activity, worse functional outcomes, and higher radiographic progression scores (Table 2). Having a flare was associated with a larger than minimal clinically important difference increase in HAQ-DI $(\beta=0.32,95 \%$ CI $0.29-0.36$; $\mathrm{p}<0.01)$ and decrease in EQ-5D $(\beta=-0.11,95 \% \mathrm{CI}-0.12$ to $-0.09 ; \mathrm{p}<0.01)$. Both the physical and mental performance measures from SF-36 were significantly worse in patients who flared in the unadjusted model. This was more marked with the PCS and did not remain significant with the MCS in the adjusted model. Patients who flared were 3.6 times $(95 \%$ CI $2.77-4.67 ; \mathrm{p}<0.01)$ more likely to have erosive progression, defined as new or larger erosions over 1 year on radiographs.

\section{DISCUSSION}

In this prospective study, one-third of RA patients with LDA states experienced a flare during 12 months of followup. This is similar to flare rates reported in cohort studies, although these included only patients in remission ${ }^{5,9}$ and in drug tapering studies in patients who remain on stable therapy. In both the DRESS ${ }^{23}$ and the POET ${ }^{24}$ studies, the rate of short-lived flare was significantly higher in patients who tapered or stopped their anti-TNF therapy compared to those who continued treatment, although in the DRESS study, the rate of major flares was similar between the 2 groups.

In our study, we have shown that the occurrence of a flare is hard to predict, but undeniably associated with worse clinical outcomes at 12 months. Our study highlights that identification of predictors of flare in patients with LDA states is challenging. In accordance with a previous remission cohort study ${ }^{9}$, we found that HAQ-DI, a measure of functional activity, reflected by difficulties in activities of daily living, was predictive for flare. It is plausible that patients with LDA and high functional disability are more likely to flare. Functional impairment can herald a flare with the onset of morning stiffness and fatigue. A high HAQ may reflect severe rheumatoid arthritis with disease-related damage and the likelihood of grumbling disease (persistent low-grade disease activity).

Serum biomarkers were only modestly correlated with DAS28 at the time of flare. This might be because a flare is defined by worsening of the DAS28 composite score, and an increase in TJC and PtGA alone may increase the DAS28 
Table 2. Outcomes at 1 year in patients who flare compared to patients who do not flare.

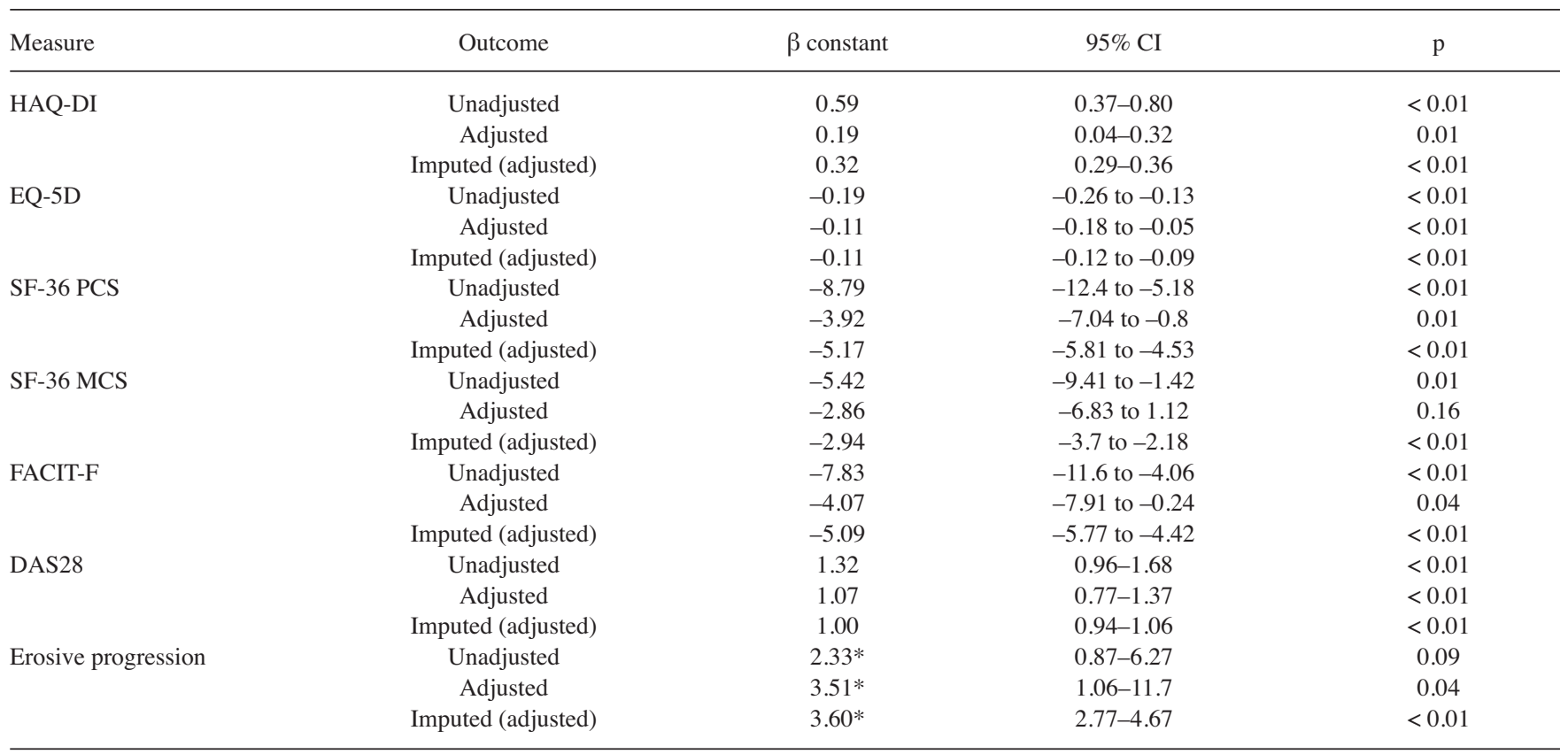

* OR values. HAQ-DI: Health Assessment Questionnaire-Disability Index; SF-36 PCS: Medical Outcomes Study Short Form-36 physical component summary; MCS: mental component summary; FACIT-F: Functional Assessment of Chronic Illness Therapy Fatigue scale; DAS28: 28-joint count Disease Activity Score.

score to a sufficient level to define a flare. It is possible that a flare event is not solely the result of direct synovial inflammation but may be driven by other pathways, for example chronification of pain due to central sensitization and abnormal regulatory mechanisms ${ }^{25}$. This heterogeneity may partly explain why identifying predictors of flare is challenging. The OMERACT RA flare group recognizes the limitation of DAS28 in defining flare events. They are developing a consensus-based core domain set to identify and measure flare in $\mathrm{RA}^{26,27}$. It is likely that improving the definition of flare and establishing a scoring system may help interpret predictors of flare in the future.

We found that a higher baseline CRP and ESR were predictive of flare in the univariate analyses, while baseline MBDA score, calprotectin, and CXCL10 were not. In the sensitivity analysis limited to flare events with an associated high MBDA score at the time of flare, a relationship between baseline MBDA value and flare risk was established. This may suggest that baseline MBDA score is only predictive of flares that are driven directly by inflammation. Interestingly, when each component of the MBDA score was analyzed individually, only 3 of the 12 components (SAA, leptin, and high-sensitivity CRP) predicted flare. Studies suggest a close correlation between leptin levels and RA disease duration, activity, and severity ${ }^{28}$. The rapid production of SAA and its exceptionally wide dynamic range has proved advantageous as a biomarker of disease activity, with superiority over CRP in early RA studies ${ }^{29}$.

US variables, including PD signal, had no predictive value in our study. This is likely a reflection of the high proportion of patients in our cohort who had US activity at baseline. In the POET study, only $63 \%$ of patients had US signs of arthritis with positive PD signal ${ }^{30}$. This is partly explained by our cohort, which included a greater proportion of patients with LDA states above the DAS28 remission cutoff. A large number of patients were taking DMARD monotherapy, and only 3 were prescribed oral corticosteroids, which may explain the difference in PD compared to other cohorts that have achieved LDA states with combination DMARD and corticosteroid therapy. Scoring of PD was also more stringent in our cohort compared to others ${ }^{9}$, leading to a much higher proportion of patients with PD signal being reported. The major limitation of US is that it remains a user-dependent technique. It is increasingly sensitive at demonstrating evidence of incomplete suppression of inflammation. The joints of healthy volunteers have been shown to display PD signal $^{31,32}$, and treatment escalation studies have argued against very stringent US targets ${ }^{33}$. Others have also shown that low-grade PD signal and synovial hypertrophy may not necessarily reflect the presence of active synovitis in RA joints ${ }^{34}$. In our cohort, a high proportion had PD activity at baseline and did not go on to flare. It may be postulated that a binary PD cutoff might be insensitive in discriminating patients who are likely to flare.

Our study also found that patients who flare were more likely to have erosive progression, worse quality of life, and higher disease activity over 1 year. These findings are consistent with previous studies ${ }^{7,9,35}$ and emphasize the

Personal non-commercial use only. The Journal of Rheumatology Copyright @ 2018 . All rights reserved. 
importance of flare and its relationship with patient outcomes. What remains unclear is whether flares are causally implicated in clinical outcome or if they are merely a biomarker of persistent low-grade disease. A flare may imply persistent uncontrolled inflammation contributing to disease progression or a transient episode of inflammation (e.g., a 6-week flare within a stable 6-month period) that is sufficient to affect longterm outcome, or signify negative patient experience, and a lack of self-control and unpredictability of the disease, which undoubtedly have psychological health implications.

There were several strengths of our study. The cohort was selected from routine care, which is far more representative than a highly selective clinical trial population. Using patients in LDA states rather than remission enables access to a broader range of patients and is more in keeping with routine clinical care. Further, this was a deeply phenotyped cohort with extensive clinical and laboratory data at multiple timepoints across the study period.

There are potential limitations to our study. We must acknowledge the limitation of the REMIRA study sample size, and the limited number of predictors identified could reflect a type 2 error. We also acknowledge issues with missing data, particularly with incomplete available US reports. However, the pattern of missing data met the assumptions of missing at random and we were able to successfully construct an imputation model to address this. We only registered flares during a visit to a rheumatologist and the actual flare rate might be higher. Potential flares between visits could have been detected by a flare questionnaire ${ }^{36}$ or alternative tools that permit remote monitoring. However, we would have missed only short-lived flares $(<3 \mathrm{mos})$, and those are of less clinical importance because they are less likely to lead to worse clinical outcomes (e.g., no radiographic progression ${ }^{23}$. REMIRA was an observational study and any modifications in medications were carried out according to the physicians' and patients' choices. Because treatment was not protocolized, this may have affected the rate of flares. A single failure model was used to identify predictors of flare, and thus changes in therapy after a flare event should not influence the analysis. It is, however, possible that treatment modifications, for example, glucocorticoids during a flare, may improve disease outcome at 12 months.

We have demonstrated that flares are common in RA patients with LDA states and are strongly associated with poor clinical outcomes. Therefore, preventing flares is clinically relevant yet relatively challenging. HAQ-DI, a measure of functional activity, was an important predictor of flare. However, flares are complex events and not simply a reflection of inflammatory disease activity. It is possible that 2 distinct subtypes of flare might exist: an "inflammatory" flare predominately driven by an increase in SJC and ESR, and a "noninflammatory" flare with a disproportionately elevated TJC and a high PtGA score. Differentiating these 2 flare types may identify potential predictors. Further research is needed to determine whether distinct flares exist and to categorize the potential predictors of each.

\section{ACKNOWLEDGMENT}

We acknowledge the Crescendo Bioscience team, in particular Eric Sasso and Nadine Defranoux, for processing the REMIRA blood samples and assisting in the completion of this manuscript. We also acknowledge Dr. Stephen Kelly for his advice on setting up the US protocols.

\section{REFERENCES}

1. Singh JA, Saag KG, Bridges SL Jr., Akl EA, Bannuru RR, Sullivan $\mathrm{MC}$, et al; American College of Rheumatology. 2015 American College of Rheumatology guideline for the treatment of rheumatoid arthritis. Arthritis Care Res 2016;68:1-25.

2. Smolen JS, Aletaha D, Bijlsma JW, Breedveld FC, Boumpas D, Burmester G, et al; T2T Expert Committee. Treating rheumatoid arthritis to target: recommendations of an international task force. Ann Rheum Dis 2010;69;631-7.

3. Bingham CO 3rd, Pohl C, Woodworth TG, Hewlett SE, May JE, Rahman MU, et al. Developing a standardized definition for disease "flare" in rheumatoid arthritis (OMERACT 9 Special Interest Group). J Rheumatol 2009;36:2335-41.

4. Alten R, Pohl C, Choy EH, Christensen R, Furst DE, Hewlett SE, et al; OMERACT RA Flare Definition Working Group. Developing a construct to evaluate flares in rheumatoid arthritis: a conceptual report of the OMERACT RA Flare Definition Working Group. J Rheumatol 2011;38:1745-50.

5. Molenaar ET, Voskuyl AE, Dinant HJ, Bezemer PD, Boers M, Dijkmans BA. Progression of radiologic damage in patients with rheumatoid arthritis in clinical remission. Arthritis Rheum 2004;50:36-42

6. Welsing PM, Landewe RB, van Riel PL, Boers M, van Gestel AM, van der Linden $\mathrm{S}$, et al. The relationship between disease activity and radiologic progression in patients with rheumatoid arthritis: a longitudinal analysis. Arthritis Rheum 2004;50:2082-93.

7. Markusse IM, Dirven L, Gerards AH, van Groenendael JH, Ronday HK, Kerstens PJ, et al. Disease flares in rheumatoid arthritis are associated with joint damage progression and disability: 10-year results from the BeSt study. Arthritis Res Ther 2015;17:232.

8. Myasoedova E, Chandran A, Ilhan B, Major BT, Michet CJ, Matteson EL, et al. The role of rheumatoid arthritis (RA) flare and cumulative burden of RA severity in the risk of cardiovascular disease. Ann Rheum Dis 2016;75:560-5.

9. Saleem B, Brown AK, Quinn M, Karim Z, Hensor EM, Conaghan P, et al. Can flare be predicted in DMARD treated RA patients in remission, and is it important? A cohort study. Ann Rheum Dis 2012;71:1316-21.

10. Han J, Geng Y, Deng X, Zhang Z. Subclinical synovitis assessed by ultrasound predicts flare and progressive bone erosion in rheumatoid arthritis patients with clinical remission: a systematic review and metaanalysis. J Rheumatol 2016;43:2010-8.

11. Bouman CA, van der Maas A, van Herwaarden N, Sasso EH, van den Hoogen FH, den Broeder AA. A multi-biomarker score measuring disease activity in rheumatoid arthritis patients tapering adalimumab or etanercept: predictive value for clinical and radiographic outcomes. Rheumatology 2017;56:973-80.

12. Nordal HH, Brokstad KA, Solheim M, Halse AK, Kvien TK, Hammer HB. Calprotectin (S100A8/A9) has the strongest association with ultrasound-detected synovitis and predicts response to biologic treatment: results from a longitudinal study of patients with established rheumatoid arthritis. Arthritis Res Ther 2017;19:3.

Personal non-commercial use only. The Journal of Rheumatology Copyright @ 2018 . All rights reserved. 
13. Patro PS, Singh A, Misra R, Aggarwal A. Myeloid-related protein 8/14 levels in rheumatoid arthritis: marker of disease activity and response to methotrexate. J Rheumatol 2016;43:731-7.

14. Nordal HH, Brun JG, Hordvik M, Eidsheim M, Jonsson R, Halse AK. Calprotectin (S100A8/A9) and S100A12 are associated with measures of disease activity in a longitudinal study of patients with rheumatoid arthritis treated with infliximab. Scand J Rheumatol 2016;45:274-81.

15. Choi IY, Gerlag DM, Herenius MJ, Thurlings RM, Wijbrandts CA, Foell D, et al. MRP8/14 serum levels as a strong predictor of response to biological treatments in patients with rheumatoid arthritis. Ann Rheum Dis 2015;74:499-505.

16. Pandya JM, Lundell AC, Andersson K, Nordstrom I, Theander E, Rudin A. Blood chemokine profile in untreated early rheumatoid arthritis: CXCL10 as a disease activity marker. Arthritis Res Ther 2017;19:20

17. Ma MHY, Ibrahim F, Kingsley GH, Cope A, Scott DL. Variable impacts of different remission states on health-related quality of life in rheumatoid arthritis. Clin Exp Rheumatol 2018;36:203-9.

18. van der Maas A, Lie E, Christensen R, Choy E, de Man YA, van Riel P, et al. Construct and criterion validity of several proposed DAS28-based rheumatoid arthritis flare criteria: an OMERACT cohort validation study. Ann Rheum Dis 2013;72:1800-5.

19. Centola M, Cavet G, Shen Y, Ramanujan S, Knowlton N, Swan KA, et al. Development of a multi-biomarker disease activity test for rheumatoid arthritis. PLoS One 2013;8:e60635.

20. Ohrndorf S, Backhaus M. Advances in sonographic scoring of rheumatoid arthritis. Ann Rheum Dis 2013;72:ii69-75.

21. Wakefield RJ, Balint PV, Szkudlarek M, Filippucci E, Backhaus M, D'Agostino MA, et al; OMERACT 7 Special Interest Group. Musculoskeletal ultrasound including definitions for ultrasonographic pathology. J Rheumatol 2005;32:2485-7.

22. Swinscow TD, Campbell MJ. Chapter 11: correlation and regression. In: Statistics at square one. London: BMJ Publishing Group; 1997.

23. van Herwaarden N, van der Maas A, Minten MJ, van den Hoogen FH, Kievit W, van Vollenhoven RF, et al. Disease activity guided dose reduction and withdrawal of adalimumab or etanercept compared with usual care in rheumatoid arthritis: open label, randomised controlled, non-inferiority trial. BMJ 2015;350:h1389.

24. Ghiti Moghadam M, Vonkeman HE, Ten Klooster PM, Tekstra J, van Schaardenburg D, Starmans-Kool M, et al; Dutch National POET Collaboration. Stopping tumor necrosis factor inhibitor treatment in patients with established rheumatoid arthritis in remission or with stable low disease activity: a pragmatic multicenter, open-label randomized controlled trial. Arthritis Rheumatol 2016;68:1810-7.
25. Schaible HG, von Banchet GS, Boettger MK, Bräuer R, Gajda M, Richter F, et al. The role of proinflammatory cytokines in the generation and maintenance of joint pain. Ann N Y Acad Sci 2010;1193:60-9.

26. Bykerk VP, Lie E, Bartlett SJ, Alten R, Boonen A, Christensen R, et al. Establishing a core domain set to measure rheumatoid arthritis flares: report of the OMERACT 11 RA flare workshop. J Rheumatol 2014;41:799-809.

27. Bykerk VP, Bingham CO, Choy EH, Lin D, Alten R, Christensen R, et al. Identifying flares in rheumatoid arthritis: reliability and construct validation of the OMERACT RA Flare Core Domain Set. RMD Open 2016;2:e00225.

28. Abella V, Scotece M, Conde J, Pino J, Gonzalez-Gay MA, Gómez-Reino JJ et al. Leptin in the interplay of inflammation, metabolism and immune system disorders. Nat Rev Rheumatol 2017;13:100-9

29. Hwang YG, Balasubramani GK, Metes ID, Levesque MC, Bridges SL Jr, Moreland LW. Differential response of serum amyloid A to different therapies in early rheumatoid arthritis and its potential value as a disease activity biomarker. Arthritis Res Ther 2016;18:108.

30. Lamers-Karnebeek FB, Luime JJ, Ten Cate DF, Teerenstra S, Swen NW, Gerards AH, et al. Limited value for ultrasonography in predicting flare in rheumatoid arthritis patients with low disease activity stopping TNF inhibitors. Rheumatology 2017;56:1560-5.

31. Ellegaard K, Torp-Pedersen S, Holm CC, Danneskiold-Samsøe B, Bliddal H. Ultrasound in finger joints: findings in normal subjects and pitfalls in the diagnosis of synovial disease. Ultraschall Med 2007;28:401-8.

32. Terslev L, Torp-Pedersen S, Qvistgaard E, von der Recke P, Bliddal H. Doppler ultrasound findings in healthy wrists and finger joints. Ann Rheum Dis 2004;63:644-8.

33. Dale J, Stirling A, Zhang R, Purves D, Foley J, Sambrook M, et al. Targeting ultrasound remission in early rheumatoid arthritis: the results of the TaSER study, a randomised clinical trial. Ann Rehum Dis 2016;75:1043-50.

34. Gartner M, Mandl P, Radner H, Supp G, Machold KP, Aletaha D, et al. Sonographic joint assessment in rheumatoid arthritis: associations with clinical joint assessment during a state of remission. Arthritis Rheum 2013;65;2005-14.

35. Ometto F, Raffeiner B, Bernardi L, Bostsios C, Veronese N, Punzi $\mathrm{L}$, et al. Self-reported flares are predictors of radiographic progression in rheumatoid arthritis patients in 28-joint disease activity score remission: a 24-month observational study. Arthritis Res Ther 2016;18:89

36. Bykerk VP, Shadick N, Frits M, Bingham CO, Jeffery I, Iannaccone C. Flares in rheumatoid arthritis: frequency and management. A report from the BRASS registry. J Rheumatol 2014;41:227-34. 